\title{
RESOLVING THE PLANET-HOSTING INNER REGIONS OF THE LkCa 15 DISK*
}

C. Thalmann $^{1}$, M. Janson ${ }^{2}$, A. Garufi ${ }^{1,3,4}$, A. Boccaletti ${ }^{5}$, S. P. Quanz ${ }^{1}$, E. Sissa ${ }^{6,7}$, R. Gratton ${ }^{6}$, G. Salter ${ }^{8}$, M. Benisty ${ }^{9,10}$, M. Bonnefoy ${ }^{9,10}$, G. Chauvin ${ }^{9,10}$, S. Daemgen ${ }^{1}$, S. Desidera ${ }^{6}$, C. Dominik ${ }^{11}$, N. Engler ${ }^{1}$, M. Feldi $^{12}$,

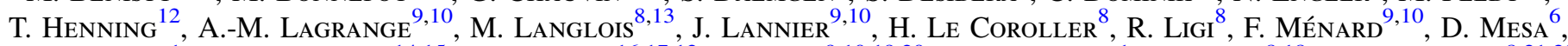
M. R. Meyer ${ }^{1}$, G. D. Mulders ${ }^{14,15}$, J. Olofsson ${ }^{16,17,12}$, C. Pinte ${ }^{9,10,19,20}$, H. M. Schmid ${ }^{1}$, A. Vigan ${ }^{8,18}$, and A. Zurlo ${ }^{8,21,22}$

${ }^{1}$ ETH Zurich, Institute for Astronomy, Wolfgang-Pauli-Strasse 27, 8093 Zurich, Switzerland; thalmann@phys.ethz.ch

${ }^{2}$ Department of Astronomy, Stockholm University, SE-106 91 Stockholm, Sweden

${ }^{3}$ Universidad Autonónoma de Madrid, Departamento de Física Teórica, Módulo 15, Facultad de Ciencias, Campus de Cantoblanco, E-28049 Madrid, Spain ${ }^{4}$ Unidad Asociada CAB-UAM, Madrid, Spain

${ }^{5}$ LESIA, Observatoire de Paris, PSL Research University, CNRS, Université Paris Diderot, Sorbonne Paris Cité, UPMC Paris 6, Sorbonne Université, 5 place Jules Janssen, F-92195 Meudon CEDEX, France

6 INAF-Osservatorio Astronomico di Padova, Vicolo dell'Osservatorio 5, I-35122 Padova, Italy

7 Dipartimento di Fisica e Astronomia "G. Galilei," Università degli Studi di Padova, Vicolo dell'Osservatorio 3, I-35122 Padova, Italy

${ }^{8}$ Aix Marseille Université, CNRS, LAM (Laboratoire d'Astrophysique de Marseille) UMR 7326, F-13388 Marseille, France

${ }^{9}$ Université Grenoble Alpes, IPAG, F-38000 Grenoble, France

${ }^{10}$ CNRS, IPAG, F-38000 Grenoble, France

${ }^{11}$ Anton Pannekoek Institute, University of Amsterdam, Science Park 904, 1098 XH Amsterdam, The Netherlands

${ }^{12}$ Max-Planck-Institut für Astronomie, Königstuhl 17, D-69117 Heidelberg, Germany
${ }^{13}$ CRAL, UMR 5574, CNRS, Université Lyon 1, 9 avenue Charles André, F-69561, Saint Genis Laval Cedex, France

${ }^{14}$ Lunar and Planetary Laboratory, The University of Arizona, Tucson, AZ 85721, USA

${ }^{15}$ Earths in Other Solar Systems Team, NASA Nexus for Exoplanet System Science, Tucson, AZ, USA

${ }^{16}$ Instituto de Física y Astronomía, Facultad de Ciencias, Universidad de Valparaíso, Av. Gran Bretaña 1111, Playa Ancha, Valparaíso, Chile

${ }^{17}$ ICM nucleus on protoplanetary disks, Universidad de Valparaíso, Av. Gran Bretaña 1111, Valparaíso, Chile

${ }^{18}$ European Southern Observatory, Alonso de Cordova 3107, Vitacura, Santiago, Chile

${ }^{19}$ UMI-FCA, CNRS/INSU (UMI 3386), Paris, France

${ }^{20}$ Departamento de Astronomía, Universidad de Chile, Santiago, Chile

${ }^{21}$ Núcleo de Astronomía, Facultad de Ingeniería, Universidad Diego Portales, Av. Ejercito 441, Santiago, Chile

${ }^{22}$ Millennium Nucleus "Protoplanetary Disk," Departamento de Astronomía, Universidad de Chile, Casilla 36-D, Santiago, Chile Received 2016 July 7; revised 2016 August 24; accepted 2016 August 24; published 2016 September 9

\begin{abstract}
LkCa 15 hosts a pre-transitional disk as well as at least one accreting protoplanet orbiting in its gap. Previous disk observations have focused mainly on the outer disk, which is cleared inward of $\sim 50$ au. The planet candidates, on the other hand, reside at orbital radii around 15 au, where disk observations have been unreliable until recently. Here, we present new $J$-band imaging polarimetry of LkCa 15 with SPHERE IRDIS, yielding the most accurate and detailed scattered-light images of the disk to date down to the planet-hosting inner regions. We find what appear to be persistent asymmetric structures in the scattering material at the location of the planet candidates, which could be responsible at least for parts of the signals measured with sparse-aperture masking. These images further allow us to trace the gap edge in scattered light at all position angles and search the inner and outer disks for morphological substructure. The outer disk appears smooth with slight azimuthal variations in polarized surface brightness, which may be due to shadowing from the inner disk or a two-peaked polarized phase function. We find that the near-side gap edge revealed by polarimetry matches the sharp crescent seen in previous ADI imaging very well. Finally, the ratio of polarized disk to stellar flux is more than six times larger in the $J$-band than in the $R I$ bands.
\end{abstract}

Key words: circumstellar matter - planets and satellites: formation - protoplanetary disks - stars: individual (LkCa 15) - stars: pre-main sequence - techniques: high angular resolution

\section{INTRODUCTION}

Transitional disks have properties that can be interpreted as intermediate between the protoplanetary and debris disk phases, with an optically thick outer disk and typically a wide (tens of au) gap within (e.g., Strom et al. 1989; Calvet et al. 2005). One possible origin for such gaps is dynamical clearing by forming planets. The transitional disk host LkCa 15 has emerged as a benchmark target for this scenario. LkCa 15 is a K5-type star at a distance of $\sim 140 \mathrm{pc}$ located in the TaurusAuriga star-forming region (Simon et al. 2000). Resolved imaging ranging from the visible to millimeter wavelengths

\footnotetext{
Based on data collected at the European Southern Observatory, Chile (ESO Programme 096.C-0248(A)).
}

(e.g., Andrews et al. 2011; Isella et al. 2014; Thalmann et al. 2014) confirmed the morphological structure implied from spectral energy distribution fitting (Espaillat et al. 2007), all showing an outer disk truncated inward of $\sim 50$ au. Recent scattered-light imaging also revealed an inner disk component within the gap structure (Thalmann et al. 2015; Oh et al. 2016).

LkCa 15 stands out in that directly imaged planet candidates have been reported in the system. The first candidate was published by Kraus \& Ireland (2012), and subsequently two more candidates were proposed by Sallum et al. (2015). We follow the three-object nomenclature of Sallum et al. (2015), in which "b" refers to a candidate at $\sim 14.7$ au that displays $\mathrm{H} \alpha$ emission, with " $\mathrm{c}$ " and "d" following counterclockwise. The $\mathrm{H} \alpha$ emission of "b" makes a particularly compelling case for a protoplanet, as it implies active gas accretion. 
Here, we present new deep, high-contrast images of LkCa 15 with Spectro-polarimetric High-contrast Exoplanet REsearch (SPHERE; Beuzit et al. 2008), offering the first full map of the gap's outer edge and revealing the inner disk structures hinted at by earlier studies. These images also serve to complement the observations in which the planet candidates were discovered, where the precise morphology of the emission cannot be uniquely determined (with the exception of the $\mathrm{H} \alpha$ imaging), since they are based on sparse-aperture masking (SAM) and thus do not fully sample the pupil plane of the telescope.

\section{OBSERVATIONS AND DATA REDUCTION}

On 2015 December 19, we obtained two sets of imaging polarimetry data with the IRDIS branch (Dohlen et al. 2008; Langlois et al. 2014) of SPHERE at the European Southern Observatory's Very Large Telescope. The extreme adaptive optics (SAXO; Fusco et al. 2006) yielded a point-spread function (PSF) with an FWHM of $\sim 4$ pixels ( $\approx 50$ mas) in the broadband $J$ filter (BB_J, $1.26 \mu \mathrm{m}$ ) under good conditions (seeing 0 ". $6-0$ ". 8 in the $V$-band).

The first observation, hereafter named DEEP, aimed at maximizing sensitivity for the faint disk structures and therefore employed the N_ALC_YJ_S coronagraph (apodized Lyot, inner working angle 80 mas) with long exposures (32 s). The observation comprised 15 polarimetric cycles of 2 exposures at each of the four standard half-wave plate positions for a total of $3840 \mathrm{~s}$ of exposure.

The second observation, FAST, was executed immediately afterward without coronagraph and with minimal exposure times $(0.85 \mathrm{~s})$ to leave the star unsaturated and grant access to the innermost regions. Three polarimetric cycles with 40 exposures per position yielded $102 \mathrm{~s}$ of exposure.

Frame registration was performed on FAST by fitting twodimensional Gaussians to the unsaturated target star in each frame. Since those measured star locations appeared stable (jitter $\sim 2$ mas) with no visible drift, we adopted their median star location for all DEEP frames. Apart from removing the coronagraph, no changes were made to the instrument setup between DEEP and FAST runs.

The data were reduced using custom IDL routines implementing the double-ratio polarimetry and empirical correction of instrumental and stellar polarization from Avenhaus et al. (2014b). We present the results as polarcoordinate Stokes components $\left(Q_{\phi}, U_{\phi}\right)$, in which positive $Q_{\phi}$ signals correspond to azimuthally oriented linear polarization (Quanz et al. 2013; Avenhaus et al. 2014b; Thalmann et al. 2015). In face-on or mildly inclined circumstellar disks, this convention maps almost all scattered light into a positive $Q_{\phi}$ signal. However, due to LkCa 15's inclination of $50^{\circ}$ (Thalmann et al. 2014), multiple-scattering events also produce faint $U_{\phi}$ signals (Canovas et al. 2015). $U_{\phi}$ therefore provides an upper limit for the noise level.

Furthermore, on 2015 November 29, we obtained nonpolarimetric imaging, hereafter named FULL, with SPHERE IRDIS in dual-band imaging mode (DBI; Vigan et al. 2010) with the $\mathrm{K} 1 \mathrm{~K} 2$ filter $\left(\lambda_{\mathrm{K} 1}=2.11 \mu \mathrm{m}, \lambda_{\mathrm{K} 2}=2.25 \mu \mathrm{m}\right)$ as part of the SpHere INfrared survey for Exoplanets (SHINE; G. Chauvin et al. 2016, in preparation). Pupil tracking was used to allow for angular differential imaging (ADI; Marois et al. 2006). We obtained a sequence of $4288 \mathrm{~s}$ totaling a field rotation of 24.5 , using the N_ALC_YJH_S coronagraph (apodized Lyot, inner working angle 93 mas). Non-coronagraphic frames were obtained before and after the coronagraphic sequence for photometric calibration. All data were reduced with the SPHERE pipeline (Pavlov et al. 2008) and additional analysis tools implemented at the SPHERE data center, including ADI with KLIP (five subtracted modes, 24.5 field rotation; Soummer et al. 2012; Pueyo et al. 2015). However, since these observations are less sensitive to planets than previous studies, we only use them to complement our disk analysis (see Section 3.3).

\section{RESULTS AND DISCUSSION}

\subsection{Imagery}

Our $J$-band polarimetry of LkCa 15 is shown in Figure 1. The DEEP $Q_{\phi}$ images (Figure 1(a)) reveal the inner and outer disk components and the gap between them at much higher signal-to-noise ratio $(\mathrm{S} / \mathrm{N})$ than previously achieved (Thalmann et al. 2015). To quantify the detection, we calculate $S / N$ maps (Figure 1(b)) by dividing the $Q_{\phi}$ and $U_{\phi}$ images by a radial noise profile obtained by calculating the standard deviation of pixel values in concentric annuli in the $U_{\phi}$ image. Both disk components are detected at high significance in $Q_{\phi}$, with local $\mathrm{S} / \mathrm{N}$ exceeding $5 \sigma$ over an area of $0.23 \operatorname{arcsec}^{2}$ and peaking at $11 \sigma$. Finally, we present the $Q_{\phi}$ and $U_{\phi}$ images after scaling with an $r^{2}$ map, where $r$ represents the estimated physical distance of each pixel's scattering material from the star, assuming it lies on the disk's inclined midplane $\left(50^{\circ}\right.$; Figure 1(c)). This counteracts the $r^{-2}$ dependency of the scattered-light intensity and thus reduces the dynamic range of the image, rendering disk components at different separations directly comparable. This reveals the inner edge of the outer disk as a sharp intensity step ( $60 \%$ over 1 FWHM; cf. Section 3.4) in $Q_{\phi}$, allowing us to trace the edge at all position angles for the first time. The residuals in the $U_{\phi}$ image appear as a faint quadrupole pattern diagonal to the principal axes of the projected disk, as expected from higher-order scattering (Canovas et al. 2015). The brightest structures in $r^{2}$-scaled $U_{\phi}$ are $\sim 4$ times fainter than those in $r^{2}$-scaled $Q_{\phi}$.

Figures 1(d)-(f) show the same images for FAST. Despite the lower sensitivity, the same structures as in DEEP can be discerned. The calculated $\mathrm{S} / \mathrm{N}$ drops sharply within a radius of $\sim 80$ mas, though this could be due to astrophysical signal from higher-order scattering dominating the $U_{\phi}$ image at those separations, causing the noise to be overestimated. For now, we assume that FAST does not detect the disk at smaller separations than DEEP. However, the disk structures appear notably sharper in FAST. This may be due to the fact that those were registered on a frame-to-frame basis, whereas the long exposure times and global registration of DEEP may have introduced some smearing.

\subsection{Inner Disk Structure and Planet Candidates}

Whereas the imagery in Thalmann et al. (2015) and Oh et al. (2016) sufficed to discover and confirm the presence of scattered light from the inner disk, our new data now allow us to investigate its morphology. Figure 2 shows a comparison of the inner regions of the $r^{2}$-scaled $Q_{\phi}$ images from DEEP (panel (a)) and FAST (panels (b), (c)) as well as our previously published RI-band data (panel (d); Thalmann et al. 2015).

All three images show the inner disk in strikingly similar morphology. The polarized flux is contained in a roughly 


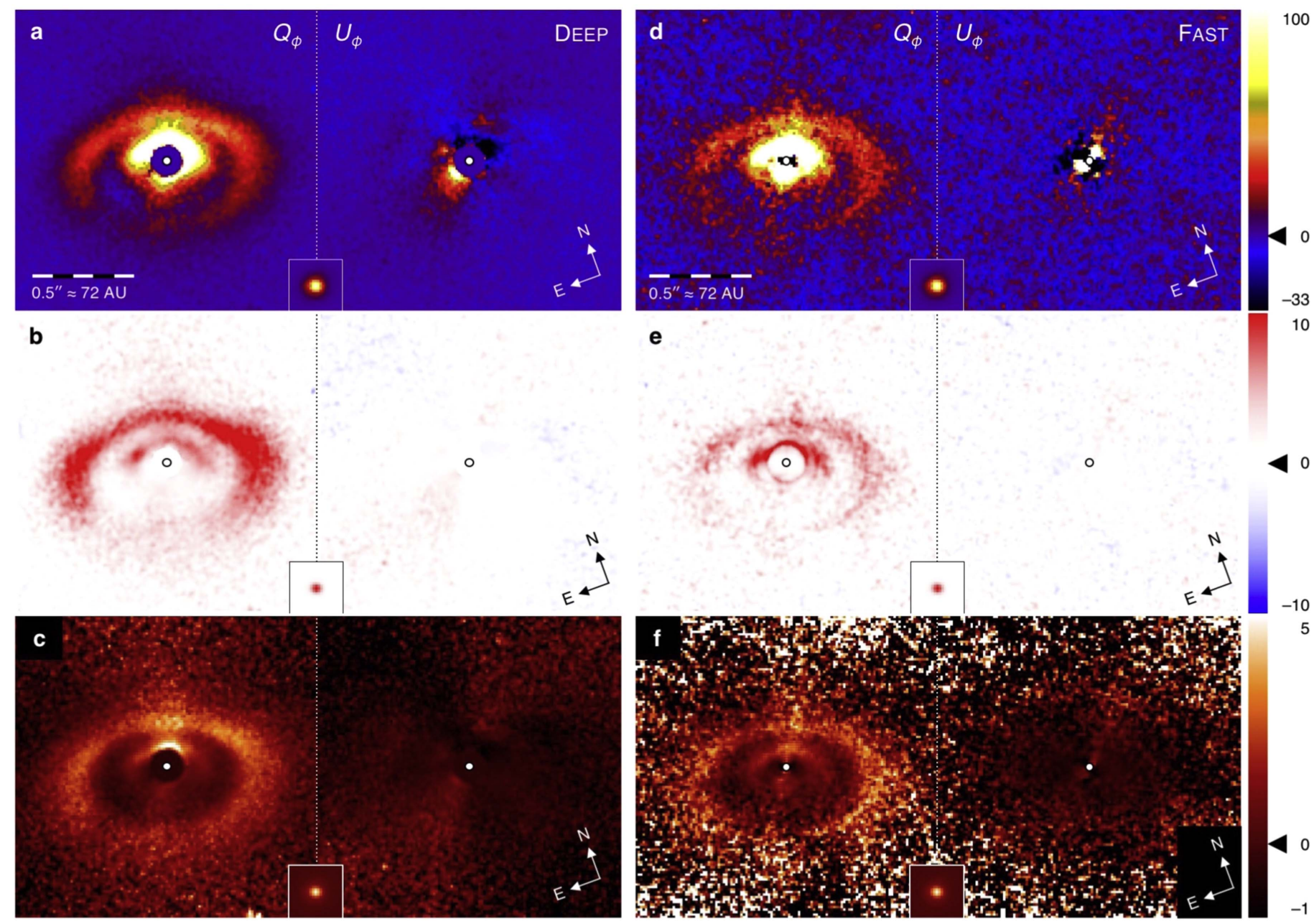

Figure 1. SPHERE IRDIS $J$-band imaging polarimetry of LkCa 15. Each panel shows the $Q_{\phi}$ and $U_{\phi}$ images side-by-side at the same scale, with insets showing the shape of the PSF core. (a) Polarized flux of DEEP at linear stretch (arb. units). The inner disk saturates the color scale. (b) The corresponding S/N map at a stretch of $[-10 \sigma, 10 \sigma]$. (c) Polarized flux of DEEP after scaling with an inclined $r^{2}$ map to render the faint disk structures visible (arb. units). ((d)-(f)) The same three images for FAST. While overall sensitivity is lower in these data, they afford an unobstructed view onto the inner disk. In all panels, the star's location is marked with a white disk. The black wedges on the color scales mark the zero level.

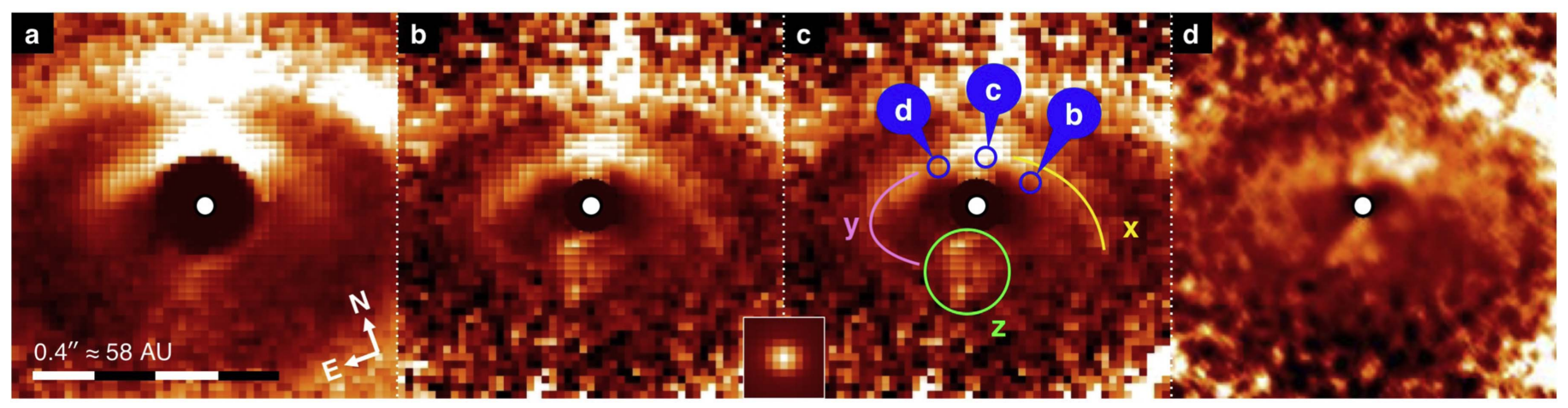

Figure 2. Structures in the inner disk of LkCa 15. (a) A close-in view of the $r^{2}$-scaled DEEP $Q_{\phi}$ image. The coronagraph's IWA is 0 !" 08 . (b) The same for FAST. The inner disk appears sharper than in (a), perhaps due to better image registration. (c) Panel (b) with annotated features. The dark blue circles marked "b," "c," "d" represent the positions of the three point sources reported in Sallum et al. (2015). Source "b" is the one detected in H $\alpha$ imaging. The pastel-colored markings identify potential persistent structures in the inner disk: two curved structures that may represent a disk edge or spiral arms (" $x$," " $y$ ") and a diffuse region along the minor axis ("z"). (d) The visible-light image from SPHERE ZIMPOL (Thalmann et al. 2015) for comparison, which reproduces those structures at least qualitatively. The small inset illustrates the shape of the PSF core.

elliptical area comparable in shape and orientation to the outer disk, but about half as large in each dimension. We note a number of morphological features that appear persistent between the three images, as marked in Figure 2(c): a bright crescent on the near side of the disk, reminiscent of the fullintensity scattered-light appearance of the outer disk (Thalmann et al. 2010, 2013); a slight asymmetry between the two crescent arms, with the western arm (" $x$ ") seemingly trending outward 

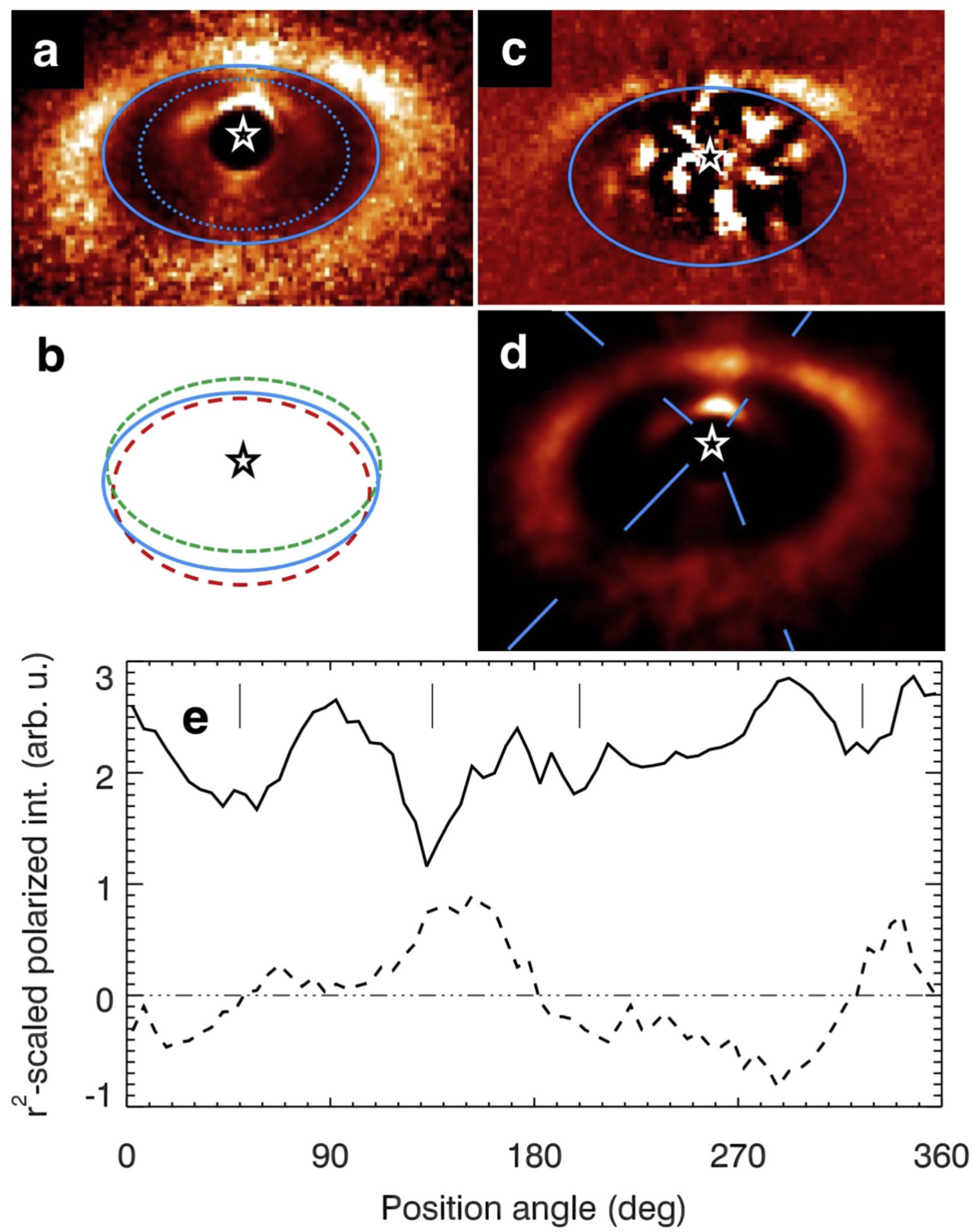

Figure 3. Analysis of the outer disk structure of LkCa 15. (a) Ellipse fits to the maximum gradient (solid blue line) and the flux minimum (dotted blue line) in the $r^{2}$ scaled DEEP $Q_{\phi}$ image. (b) Comparison of the best-fit gap edge in the $J$-band (this work; blue solid line) with those in the RI-band (Thalmann et al. 2015; red longdashed line) and submillimeter interferometry (Isella et al. 2014; green short-dashed line). (c) Full-intensity KLIP image (five subtracted modes) of the FULL data in the K1K2 filter for comparison. The gap edge derived from the $Q_{\phi}$ image coincides very well with the edge of the bright crescent in the KLIP image. (d) The image in panel (a) at a harder stretch, emphasizing the surface brightness variations in the outer disk. Four position angles with reduced brightness are marked, possibly indicating transient shadowing from the inner disk. (e) Azimuthal profile of the outer disk in $r^{2}$-scaled DEEP $Q_{\phi}$ (solid) and $U_{\phi}$ (dashed), evaluated in a 7 pixel (86 mas) wide annulus outside the best-fit gap edge at $5^{\circ}$ resolution. The four position angles from panel (d) are indicated with vertical dashes. The $U_{\phi}$ profile is dominated by the multiple-scattering quadrupole.

while the eastern arm ("y") curls inward; and a local brightening along the far side of the minor axis (" $z$ ").

Furthermore, the area within this crescent appears darkened, giving the impression of another gap in the inner disk. However, we caution that polarimetric efficiency drops in the innermost resolution elements, where the positive/negative pattern in $Q$ and $U$ is too small to be cleanly resolved (Avenhaus et al. 2014a). This could mimic a gap.

Figure 2(c) also indicates the positions of the three proposed planet candidates ("b," "c," "d"; cf. Sallum et al. 2015, LBT joint fit results), which coincide with the bright, forward-scattering side of the inner disk. The analysis and interpretation of SAM data are challenging and potentially even misleading in the presence of scattered light from a disk (e.g., Olofsson et al. 2013; Cheetham et al. 2015; Sallum et al. 2015; C. Caceres et al. 2016, in preparation); thus, the inner disk might account for some or even all of the SAM signal attributed to protoplanets. On the other hand, $\mathrm{H} \alpha$ imaging makes a strong case for a protoplanet at "b," which could be confirmed with a re-detection in $\mathrm{H} \alpha$ showing orbital motion. Such a protoplanet could be responsible for the substructure in the inner disk.

\subsection{Outer Disk Structure}

In the $r^{2}$-scaled $Q_{\phi}$ images, the outer disk appears as a diffuse region of roughly flat brightness bounded on the inside by a sharp elliptical edge. We employ the maximum merit method (Thalmann et al. 2011) to fit a parametric ellipse onto the DEEP $Q_{\phi}$ image that maximizes the brightness contrast between the annular regions immediately inside and outside of the ellipse, as detailed in Thalmann et al. (2015). We fix the position angle of the major axis to $60^{\circ}$ ( $\mathrm{cf}$. Thalmann et al. 2014). The resulting ellipse is shown in solid blue in 
Table 1

Numerical Results

\begin{tabular}{|c|c|c|c|c|c|c|}
\hline \multirow{2}{*}{\multicolumn{2}{|c|}{$\overline{\text { Outer Disk Gap Edge }}$}} & \multicolumn{2}{|l|}{$J$-band } & \multicolumn{3}{|c|}{$R I$-band } \\
\hline & & & & & & \\
\hline $\begin{array}{l}\text { Semimajor axis } \\
\quad a \text { (mas) }\end{array}$ & 367 & $(+15$ & $-25)$ & 338 & $(+11$ & $-18)$ \\
\hline -(au) & 51.5 & $(+2.1$ & $-3.4)$ & 47.4 & $(+1.5$ & $-2.5)$ \\
\hline $\begin{array}{l}\text { Semiminor axis } \\
b \text { (mas) }\end{array}$ & 235 & $(+10$ & $-15)$ & 245 & $(+21$ & $-11)$ \\
\hline -(au) & 32.9 & $(+1.4$ & $-2.1)$ & 34.3 & $(+3.0$ & $-1.5)$ \\
\hline $\begin{array}{l}\text { Major-axis off- } \\
\text { set } x \text { (mas) }\end{array}$ & -7 & $(+20$ & $-15)$ & -3 & $(+11$ & $-14)$ \\
\hline$-(\mathrm{au})$ & -1.0 & $(+2.7$ & $-2.1)$ & -0.5 & $(+1.5$ & $-2.0)$ \\
\hline $\begin{array}{l}\text { Minor-axis off- } \\
\text { set } y \text { (mas) }\end{array}$ & -51 & $(+15$ & $-10)$ & -79 & $(+11$ & $-18)$ \\
\hline$-(\mathrm{au})$ & -7.2 & $(+2.1$ & $-1.4)$ & -11.1 & $(+1.5$ & $-2.5)$ \\
\hline \multicolumn{7}{|l|}{ Gap Minimum } \\
\hline $\begin{array}{l}\text { Semimajor axis } \\
\quad a \text { (mas) }\end{array}$ & 276 & $(+92$ & $-104)$ & & & \\
\hline -(au) & 38.6 & $(+12.9$, & $-14.6)$ & & & \\
\hline $\begin{array}{l}\text { Semiminor axis } \\
\quad b \text { (mas) }\end{array}$ & 196 & $(+0$ & $-76)$ & & & \\
\hline$-(\mathrm{au})$ & 27.4 & $(+0$ & $-10.6)$ & & & \\
\hline $\begin{array}{l}\text { Major-axis off- } \\
\text { set } x \text { (mas) }\end{array}$ & 0 & $(+12$ & $-37)$ & & & \\
\hline$-(\mathrm{au})$ & 0 & $(+1.7$ & $-5.1)$ & & & \\
\hline $\begin{array}{l}\text { Minor-axis off- } \\
\text { set } y \text { (mas) }\end{array}$ & -51 & $(+27$ & $-22)$ & & & \\
\hline$-(\mathrm{au})$ & -7.2 & $(+3.8$ & $-3.1)$ & & & \\
\hline \multicolumn{7}{|c|}{ Polarized Disk/Star Contrast } \\
\hline Inner disk & & 0.0025 & & & 0.00040 & \\
\hline Outer disk & & 0.0036 & & & 0.00055 & \\
\hline $\begin{array}{l}\text { Inner/outer } \\
\text { ratio }\end{array}$ & & 0.70 & & & 0.73 & \\
\hline
\end{tabular}

Note. $J$-band results are based on this work; $R I$-band results are taken from Thalmann et al. (2015). The gap fitting parameters are explained in Section 3.3, the contrast values in Section 3.4. The accuracy of the contrast values is estimated at $\sim 10 \%$.

Figure 3(a). The fit ellipse matches the perceived gap edge accurately.

To separate the inner and outer disk for further analysis, we also fit a second ellipse along which the $r^{2}$-scaled $Q_{\phi}$ intensity is minimized. This ellipse is shown in dotted blue in Figure 3(a). The numerical parameter of both ellipses are given in Table 1. For errors, we "fit" similar ellipses to $U_{\phi}$ and measure the standard deviation of the resulting merits. The error intervals represent the family of ellipses whose $Q_{\phi}$ merits are less than one standard deviation below the best fit.

Figure 3(b) compares the gap edge fit ellipses in $J$-band polarimetry (this work; solid blue) to those in RI-band polarimetry (Thalmann et al. 2015; long-dashed red) and submillimeter interferometry (Isella et al. 2014; short-dashed green) with respect to the star's position. Our new data confirm the eccentric scattered-light gap contrasting with the symmetric gap in submillimeter thermal emission. Due to the tapered gap wall, inclination cannot explain this eccentricity (Thalmann et al. 2014), whereas shadowing from the inner disk could (Thalmann et al. 2015). The eccentricity appears slightly lower than in the RI-band. This could be due to the poorly constrained far-side edge in the $R I$-band data, color-dependent polarized reflectivity, chromatic filtering by the inner disk halo, or a
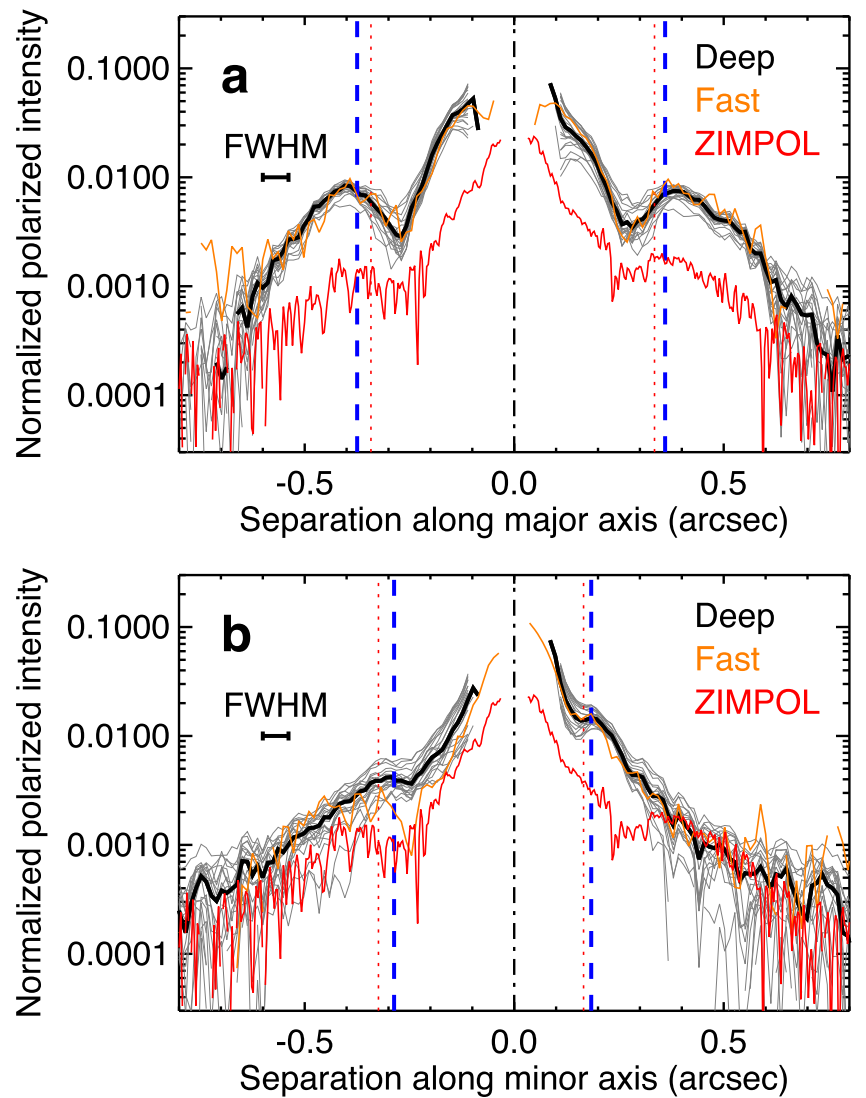

Figure 4. Polarized surface brightness profiles of the LkCa 15 disk in stellar flux per square arcsecond (see the text). (a) Profiles measured in a 86 mas wide strip along the major axis, centered on the star. Positive separations correspond to a position angle of $60^{\circ}$ on sky. (b) Profiles measured in a 257 mas wide strip along the minor axis, centered on the star. Positive separations correspond to a position angle of $330^{\circ}$. Solid black lines show the DEEP $J$-band $Q_{\phi}$ surface brightness profiles. The family of thin gray lines represents the results of adding strips from the $U_{\phi}$ image taken from different position angles to the $Q_{\phi}$ strip to illustrate the estimated noise level. Thin orange lines show the corresponding profiles from FAST, which match DEEP very well. Thin red lines represent the $R I$-band profiles from Thalmann et al. (2015) for comparison, demonstrating that the disk is significantly more reflective in polarization in $J$ band than in $R I$-band. Dashed blue vertical lines demarcate the bounds of the $J$ band gradient fit ellipse from Figure 3. Dotted red vertical lines show the corresponding bounds for the RI-band fit ellipse from Thalmann et al. (2015).

temporal evolution of the shadowing postulated as the origin of the eccentric scattered-light gap.

Figure 3(c) shows the $J$-band gap ellipse superimposed on the $K 1 K 2$-band full-intensity KLIP image of the FULL data. The ellipse coincides exactly with the sharp inner edge of the bright forward-scattering crescent, confirming the long-held assumption that such crescents in ADI images accurately trace the near-side gap edge even though the fainter parts of the disk suffer from oversubtraction effects.

The DEEP data are sensitive enough to search for substructure in the outer disk. While we find no sign of spirals or structural asymmetries, we note significant variations in the azimuthal $Q_{\phi}$ distribution. In particular, the disk appears locally dimmed in radial lanes around azimuths $50^{\circ}, 135^{\circ}, 200^{\circ}$, and $325^{\circ}$ (see Figures 3(d), (e)). Furthermore, near-side gap edge shows similar substructure in the FULL and DEEP images.

One possible scenario is that these dark lanes indicate shadows cast by inner disk regions (Marino et al. 2015; Stolker et al. 2016) or magnetospheric accretion columns (Bodman 
et al. 2016). The inner and outer disks do have similar azimuthal structure.

An alternative explanation is that a polarized scattering phase function with peaks in both the forward and backward directions (e.g., Min et al. 2016) could brighten the projected disk along its minor axis (T. Stolker et al. 2016, in preparation). A case study for this scenario is HD100546 (Garufi et al. 2016), where a bright wedge surrounded by two dark wedges was detected along the near-side minor axis. Another analogy between these two data sets is the presence of a tenuous counterpart to the bright wedge in $U_{\phi}$, which is roughly $45^{\circ}$ displaced from it. This detection may suggest small departures from centrosymmetric scattering as expected from multiple scattering in inclined disks (Canovas et al. 2015).

\subsection{Polarized Surface Brightness Profiles}

To quantify the amount of polarized light detected, we calculate surface brightness profiles in the $Q_{\phi}$ image without $r^{2}$ scaling. We extract a 86 mas wide strip along the major axis of the projected disk (position angle $60^{\circ}$; Thalmann et al. 2014) and a 257 mas wide strip along the minor axis, and average the pixel values across the width to obtain one-dimensional profiles. We then divide each profile by the sum of all pixel values in the unsaturated stellar full-intensity PSF (out to 1 !"23) $^{\prime \prime}$ and by the solid angle on sky corresponding to a pixel $\left(0{ }^{\prime \prime} 0123^{2}\right)$. The values of the resulting profiles therefore measure the local polarized flux density of the disk in units of stellar flux per square arcsecond. The resulting values do not explicitly depend on pixel size or wavelength and therefore allow for direct comparison between optical and infrared measurements. (This differs from the normalization used in Thalmann et al. 2015.)

Figures 4(a) and (b) show the profiles for the major and minor axis, respectively. The solid black curve corresponds to DEEP. To visualize the uncertainty level, we extract a number of equivalent profiles from the DEEP $U_{\phi}$ image and add them to the measured $Q_{\phi}$ profiles, yielding the family of gray curves. The thin orange curves are the $Q_{\phi}$ profiles for FAST, which match the DEEP results well. We also show the corresponding $Q_{\phi}$ profiles for the $R I$-band data from Thalmann et al. (2015) as thin red curves. While the morphology of the $R I$ - and $J$-band profiles is similar, the latter is significantly brighter overall.

We investigate this effect by summing the $Q_{\phi}$ image over the detectable parts of the inner and outer disks in each filter. We sample the inner disk from a radius of 70 mas out to the fluxminimum fit ellipse (dotted blue in Figure 3(a)), and the outer disk from that ellipse out to a radius of 1 !'225. We find that the polarized disk flux to stellar flux ratio is 6.4 times larger in $J$ than in $R I$ for the inner disk, and 6.6 times for the outer disk. The polarized flux ratio between the inner and outer disk area appears stable with wavelength $(0.70$ in $J, 0.73$ in $R I)$. Judging from the $U_{\phi}$ images, these figures are accurate on the order of $10 \%$. The numerical results are summarized in Table 1.

The photometry is likely affected by PSF-dependent loss of polarimetric sensitivity at small separations (Avenhaus et al. 2014b). However, the fact that we measure consistent color ratios for the inner and outer disks indicates that the differential effect between the two bands is small, most likely because both data sets were taken with the same telescope and AO system.
A higher polarized disk flux at longer wavelengths was also observed for the transition disk HD 135344 B by Stolker et al. (2016), who measured a factor of $\sim 2$ between the $J$ and $R I$ bands. This color factor offers an additional diagnostic for the composition of scattering dust. Our ongoing SPHERE observations are measuring polarized colors for many protoplanetary disks, which will allow for a comparative study in the near future.

This work has been carried out within the frame of the NCCR PlanetS supported by the SNSF and is supported by ANR-14-CE33-0018. ETH members acknowledge support from SNSF; INAF members from MIUR's "Progetti Premiali"; J.O. from the Millennium Nucleus RC130007 (Chilean Ministry of Economy) and ALMA/Conicyt Project 31130027. We thank the referee for insightful and constructive comments.

Facility: VLT:Melipal (SPHERE IRDIS).

\section{REFERENCES}

Andrews, S. M., Rosenfeld, K. A., Wilner, D. J., \& Bremer, M. 2011, ApJL, 742, L5

Avenhaus, H., Quanz, S. P., Meyer, M. R., et al. 2014a, ApJ, 790, 56 Avenhaus, H., Quanz, S. P., Schmid, H. M., et al. 2014b, ApJ, 781, 87 Beuzit, J.-L., Feldt, M., Dohlen, K., et al. 2008, Proc. SPIE, 7014, 701418

Bodman, E. H. L., Quillen, A. C., Ansdell, M., et al. 2016, MNRAS, submitted (arXiv:1605.03985)

Calvet, N., D’Alessio, P., Watson, D. M., et al. 2005, ApJL, 630, L185

Canovas, H., Ménard, F., de Boer, J., et al. 2015, A\&A, 582, L7

Cheetham, A., Huélamo, N., Lacour, S., de Gregorio-Monsalvo, I., \& Tuthill, P. 2015, MNRAS, 450, L1

Dohlen, K., Langlois, M., Saisse, M., et al. 2008, Proc. SPIE, 7014, 70143L Espaillat, C., Calvet, N., D’Alessio, P., et al. 2007, ApJL, 670, L135

Fusco, T., Rousset, G., Sauvage, J.-F., et al. 2006, OExpr, 14, 7515

Garufi, A., Quanz, S. P., Schmid, H. M., et al. 2016, A\&A, 588, A8

Isella, A., Chandler, C. J., Carpenter, J. M., Pérez, L. M., \& Ricci, L. 2014, ApJ, 788, 129

Kraus, A. L., \& Ireland, M. J. 2012, ApJ, 745, 5

Langlois, M., Dohlen, K., Vigan, A., et al. 2014, Proc. SPIE, 9147, 91471R

Marino, S., Perez, S., \& Casassus, S. 2015, ApJL, 798, L44

Marois, C., Correia, C., Galicher, R., et al. 2014, Proc. SPIE, 9148, 91480U

Marois, C., Lafrenière, D., Doyon, R., Macintosh, B., \& Nadeau, D. 2006, ApJ, 641, 556

Min, M., Rab, C., Woitke, P., Dominik, C., \& Ménard, F. 2016, A\&A, 585, A13

Oh, D., Hashimoto, J., Tamura, M., et al. 2016, PASJ, 68, L3

Olofsson, J., Benisty, M., Le Bouquin, J.-B., et al. 2013, A\&A, 552, A4

Pavlov, A., Feldt, M., \& Henning, T. 2008, in ASP Conf. Ser. 394, Astronomical Data Analysis Software and Systems XVII, ed. R. W. Argyle, P. S. Bunclark, \& J. R. Lewis (San Francisco, CA: ASP), 581

Pueyo, L., Soummer, R., Hoffmann, J., et al. 2015, ApJ, 803, 31

Quanz, S. P., Avenhaus, H., Buenzli, E., et al. 2013, ApJL, 766, L2

Sallum, S., Follette, K. B., Eisner, J. A., et al. 2015, Natur, 527, 342

Simon, M., Dutrey, A., \& Guilloteau, S. 2000, ApJ, 545, 1034

Soummer, R., Pueyo, L., \& Larkin, J. 2012, ApJL, 755, L28

Stolker, T., Dominik, C., Avenhaus, H., et al. 2016, arXiv:1603.00481

Strom, K. M., Strom, S. E., Edwards, S., Cabrit, S., \& Skrutskie, M. F. 1989, AJ, 97, 1451

Thalmann, C., Grady, C. A., Goto, M., et al. 2010, ApJL, 718, L87

Thalmann, C., Janson, M., Buenzli, E., et al. 2011, ApJL, 743, L6

Thalmann, C., Janson, M., Buenzli, E., et al. 2013, ApJL, 763, L29

Thalmann, C., Mulders, G. D., Hodapp, K., et al. 2014, A\&A, 566, A51

Thalmann, C., Mulders, G. D., Janson, M., et al. 2015, ApJL, 808, L41

Thalmann, C., Schmid, H. M., Boccaletti, A., et al. 2008, Proc. SPIE, 7014, $70143 \mathrm{~F}$

Vigan, A., Moutou, C., Langlois, M., et al. 2010, MNRAS, 407, 71 